PROCEEDINGS OF THE AMERICAN MATHEMATICAL SOCIETY

Volume 124, Number 11, November 1996

\title{
ISOLATED SPECTRAL POINTS
}

\author{
J. J. KOLIHA
}

(Communicated by Palle E. T. Jorgensen)

\begin{abstract}
The paper studies isolated spectral points of elements of Banach algebras and of bounded linear operators in terms of the existence of idempotents, and gives an elementary characterization of spectral idempotents. It is shown that 0 is isolated in the spectrum of a bounded linear operator $T$ if the (not necessarily closed) space $M=\left\{x: \lim _{n}\left\|T^{n} x\right\|^{1 / n}=0\right\}$ is nonzero and complemented by a closed subspace $N$ satisfying $T N \subset N \subset T X$.
\end{abstract}

\section{INTRODUCTION}

As a starting point we may consider a characterization of an isolated spectral point of a bounded linear operator $T$ on a Banach space $X$.

Proposition A. 0 is an isolated spectral point of a bounded linear operator $T$ if and only if one of the following conditions holds.

(I) $X$ is a topological direct sum $X=M \oplus N$, where $M, N$ are invariant under $T, T \mid M$ is quasinilpotent, $T \mid N$ invertible and $M \neq 0$.

(II) There is a nonzero projection $P$ commuting with $T$ such that $T P$ is quasinilpotent and $P+T$ invertible.

Condition (I) of Proposition A is applicable to operators, but would not work satisfactorily in general Banach algebras. Condition (II), studied in Section 1, does not rely on restriction of operators to invariant subspaces but on the existence of idempotents, which makes it a natural candidate for Banach algebras. It gives an elementary characterization of the spectral projection, similar to the one obtained by Harte [2]. While Condition (I) is widely used in the literature, surprisingly, Condition (II) does not seem to be well known, though it often yields considerable simplification of proofs for operators (Section 2). The invertibility of $P+T$ can be replaced by assuming that $P$ is the limit of a sequence $f_{n}(T)$ for holomorphic $f_{n}$.

Section 3 deals with bounded linear operators and with an improvement of Condition (I) to describe isolated spectral points. An explicit description of the spaces $M$ and $N$ in terms of the operator $T$ was given by Mbekhta [9, 10]. Mbekhta's sufficient condition for an isolated spectral point was improved by Schmoeger [11]. Theorems 3.2-3.4 below generalize Mbekhta's and Schmoeger's results.

By $A$ we denote a complex unital Banach algebra with unit $e$. If $a \in A, \rho(a)$, $\sigma(a), r(a)$ denote the resolvent set, spectrum and the spectral radius of $a$, respectively; iso $\sigma(a)$ and acc $\sigma(a)$ denote the set of all isolated and accumulation points of

Received by the editors February 28, 1995 and, in revised form, May 9, 1995.

1991 Mathematics Subject Classification. Primary 46H30, 47A10, 47A60.

Key words and phrases. Isolated spectral points, idempotents. 
$\sigma(a)$, repectively. The symbols $\operatorname{Inv}(A)$ and $\mathrm{qNil}(A)$ denote the set of all invertible and quasinilpotent elements of $A$, respectively. By $F(a)$ we denote the set of all elements $f(a)$ obtained from $a$ by the holomorphic functional calculus [4, Chapter VII]; $\overline{F(a)}$ is the closure of $F(a)$ in $A$. The spectral idempotent $p$ of an isolated spectral point $\mu$ of $a$ is $p=f(a)$, where $f$ is equal to 1 in a neighbourhood of $\mu$, and to 0 in a neighbourhood of $\sigma(a) \backslash\{\mu\}$.

The set of all bounded linear operators on a complex Banach space $X$ is denoted by $L(X)$. If $T \in L(X)$, then $T X$ and $T^{-1}(0)$ denote the range and the nullspace of $T$, respectively. The nullspace of $T^{n}$ is denoted by $T^{-n}(0)$.

\section{BANACH Algebras}

Throughout this section $A$ is a unital Banach algebra and $\mu$ is a complex number.

Theorem 1.1. Suppose $a, p \in A$ are such that

$$
p^{2}=p \neq 0, \quad a p=p a, \quad(a-\mu e) p \in \mathrm{qNil}(A) .
$$

Define

$$
c=c(\xi)=\xi p+a \quad(\text { for some } \xi \neq 0) .
$$

Then

$$
\sigma(c) \cup\{\mu\}=\sigma(a) \cup\{\mu+\xi\},
$$

and

$$
f(a)=f(c)(e-p)+\sum_{n=0}^{\infty} \frac{f^{(n)}(\mu)}{n !}(a-\mu e)^{n} p
$$

for any function $f$ holomorphic in a neighbourhood $\sigma(a) \cup\{\mu+\xi\}$.

Proof. Let $w=(a-\mu e) p$; then $w$ is quasinilpotent and

$$
\lambda e-a=(\lambda e-c)(e-p)+((\lambda-\mu) e-w) p .
$$

If $\lambda \notin \sigma(c) \cup\{\mu\}$, then $\lambda e-c,(\lambda-\mu) e-w$ are invertible; hence so is $\lambda e-a$ with

$$
(\lambda e-a)^{-1}=(\lambda e-c)^{-1}(e-p)+((\lambda-\mu) e-w)^{-1} p .
$$

This shows that $\sigma(a) \subset \sigma(c) \cup\{\mu\}$. Similarly,

$$
\lambda e-c=(\lambda e-a)(e-p)+((\lambda-\mu-\xi) e-w) p
$$

if $\lambda \notin \sigma(a) \cup\{\mu+\xi\}$, then $\lambda e-a,(\lambda-\mu-\xi) e-w \in \operatorname{Inv}(A)$ and so $\lambda-c \in \operatorname{Inv}(A)$. Hence $\sigma(c) \subset \sigma(a) \cup\{\mu+\xi\}$. To complete the proof of (1.3) we show that $\mu \in \sigma(a)$ and $\mu+\xi \in \sigma(c)$.

Suppose that $\mu \notin \sigma(a)$. From $(a-\mu e) p=w \in \mathrm{qNil}(A)$ and commutativity we get $(a-\mu e)^{-1} w=p \in \mathrm{qNil}(A)$. However, this contradicts $r(p)=\lim _{n \rightarrow \infty}\|p\|^{1 / n}=1$. Similarly, $(c-(\mu+\xi) e) p=w$ implies $\mu+\xi \in \sigma(c)$.

The equation (1.4) is then obtained from (1.5) by integrating around a suitable cycle. (In (1.5) we expand $((\lambda-\mu) e-w)^{-1}$ into a power series taking into account that $w$ is quasinilpotent.)

Using Theorem 1.1 we give a characterization of an isolated spectral point for an element of a Banach algebra $A$, obtaining at the same time an elementary description of a spectral idempotent. 
Theorem 1.2. A complex number $\mu$ is an isolated spectral point of $a \in A$ if and only if there exists $p \in A$ satisfying (1.1), that is,

$$
p^{2}=p \neq 0, \quad a p=p a, \quad(a-\mu e) p \in \mathrm{qNil}(A),
$$

and one of the conditions

$$
\begin{gathered}
\xi p+a-\mu e \in \operatorname{Inv}(A)(\text { for some } \xi \neq 0), \\
p \in \overline{F(a)} .
\end{gathered}
$$

The element $p$ is determined uniquely by (1.1) and by one of the conditions (1.6), (1.7); $p$ is the spectral idempotent of a corresponding to $\mu$.

Proof. Suppose first that $\mu$ is an isolated spectral point of $a$. Using the holomorphic functional calculus and the spectral mapping theorem we can verify (1.1), (1.6) and (1.7); (1.6) in fact holds for all $\xi \neq 0$.

Conversely, suppose that (1.1) holds, that $\xi \neq 0$, and define $c=\xi p+a$ as in (1.2). According to Theorem 1.1, $\mu$ is a spectral point of $a$, and $\sigma(a) \subset \sigma(c) \cup\{\mu\}$. If in addition (1.6) holds, then $\mu \in \rho(c)$ and $\mu \in$ iso $\sigma(a)$.

If (1.1) and (1.7) hold, there are $f_{n}$ such that $f_{n}(a) \rightarrow p$. Then $f_{n} \rightarrow f$ uniformly on $\sigma(a), f$ taking only the values 0 and 1 . From $\left|(\lambda-\mu) f_{n}(\lambda)\right| \leq r\left((a-\mu e) f_{n}(a)\right)$ and from the upper semicontinuity of the spectral radius it follows that $f(\lambda)=1$ if and only if $\lambda=\mu$. Let $\xi \neq 0$ and let $g_{n}(\lambda)=\xi f_{n}(\lambda)+\lambda-\mu$. Then $g_{n}(a) \rightarrow$ $\xi p+a-\mu e=c-\mu e$, while $g_{n}$ has nonzero limit on $\sigma(a)$. So $c-\mu e$ is invertible.

Finally, let $f$ be holomorphic in a neighbourhood of $\sigma(a) \cup\{\xi+\mu\}$, let $f$ be equal to 1 in a neighbourhood of $\mu$, and to 0 in a neighbourhood of $(\sigma(a) \cup\{\xi+\mu\}) \backslash\{\mu\}$. From equation (1.3) we conclude that $\sigma(c)=(\sigma(a) \cup\{\xi+\mu\}) \backslash\{\mu\}$, and so $f$ vanishes on $\sigma(c)$. By equation (1.4),

$$
f(a)=f(c)(e-p)+p=p
$$

and the result follows.

Example 1.3. Condition (1.1) guarantees that $\mu \in \sigma(a)$, but $\mu$ need not be an isolated spectral point of $a$ : Let $A=\mathbb{C} \oplus C(K)$, where $K$ is the closed unit disc. If $b(\lambda) \equiv \lambda$ on $K, p=(1,0)$ and $a=(0, b)$, then $(1.1)$ is satisfied with $\mu=0$, however, $\sigma(a)=K$.

Note 1.4. The preceding theorem is related to a result of Harte $[2,3]$ who showed the following: Let $a \in A$. Then $0 \notin \operatorname{acc} \sigma(a)$ if and only if there exists an idempotent $q \in A$ commuting with a such that $a(e-q) \in \mathrm{qNil}(A)$ and $q \in(A a) \cap(a A) ; q$ is then unique, and it is the spectral idempotent of a corresponding to $\sigma(a) \backslash\{0\}$. This follows immediately from Theorem 1.2. Indeed, write $p=e-q$. Then $e-p=u a=$ $a v$ for some $u, v \in A$, and

$$
(a+p)(u a v+p)=e+a p=(u a v+p)(a+p) ;
$$

since $a p \in \mathrm{qNil}(A)$, this implies that $a+p \in \operatorname{Inv}(A)$.

Note 1.5. Jörgens [5, Satz 4.13] proved a result for operators related to Theorem 1.2 in the case of a pole. He showed that $\mu$ is a pole of $T \in L(X)$ if and only if there is a nonzero projection $P$ such that $(T-\mu I)^{m} P=0$ for some $m$ and the operator $U=(T-\mu I)(I-P)$ has a pseudoinverse $V$ commuting with $U$ and such that $P=I-U V$. 


\section{Applichtions AND EXAmples}

First we give a simple derivation of the Laurent series in a neighbourhood of an isolated spectral point.

Example 2.1. If $\mu$ is an isolated spectral point of $a$ and $p$ the corresponding spectral idempotent, then $w=(a-\mu e) p$ is quasinilpotent and $b=p+a-\mu e$ is invertible by Theorem 1.2. For some $r>0$ and all $\lambda$ with $0<|\lambda-\mu|<r$ both $(\lambda-\mu) e-w$ and $(\lambda-\mu) e-b$ are invertible, so that

$$
\begin{aligned}
(\lambda e-a)^{-1} & =((\lambda-\mu) e-w)^{-1} p+((\lambda-\mu) e-b)^{-1}(e-p) \\
& =\sum_{n=1}^{\infty}(\lambda-\mu)^{-n} w^{n-1} p-\sum_{n=0}^{\infty}(\lambda-\mu)^{n} b^{-n-1}(e-p) \\
& =\sum_{n=1}^{\infty}(\lambda-\mu)^{-n}(a-\mu e)^{n-1} p-\sum_{n=0}^{\infty}(\lambda-\mu)^{n} g^{n+1},
\end{aligned}
$$

where $g=(p+a-\mu e)^{-1}(e-p)=b^{-1}(e-p)$ is the generalized Drazin inverse of $a-\mu e($ see $[8])$.

Examples 2.2-2.4 use Theorems 1.2 and 1.1. In each of these examples, the equivalence of conditions (i) and (ii) can be obtained by simple algebraic arguments. The equivalence of (ii) and (iii) then follows from the above mentioned theorems when we specify $\mu$ and $\xi$.

Example 2.2 $([6] \mu=1, \xi=-1)$. The following conditions on $a \in A$ are equivalent:

(i) $\left(a^{n}\right)$ converges in $A$

(ii) $a=p+c$, where $p$ is an idempotent, $c p=p c=0$ and $c^{n} \rightarrow 0$.

(iii) $\sigma(a) \subset D \cup\{1\}$, where $D$ is the open unit disc, and $\mu=1$ is a resolvent point or a simple pole of $a$.

Example 2.3 $(\mu=0, \xi=-1)$. The following conditions on $a \in A$ are equivalent:

(i) The limit $\lim _{s \rightarrow \infty} \exp (s a)$ exists in $A$.

(ii) $a=p+c$, where $p$ is an idempotent, $c p=p c=-p, \lim _{s \rightarrow \infty} \exp (s c)=0$.

(iii) $\sigma(a) \subset H \cup\{0\}$, where $H$ is the open left half plane, and $\mu=0$ is a resolvent point or a simple pole of $a$.

In (i) $\Rightarrow$ (ii) we need to show that $\lim _{s \rightarrow \infty} \exp (s a)=p$ implies $p a=0$. This follows from $\lim _{s \rightarrow \infty} s^{-1} \int_{0}^{s} \exp (t a) d t=p$ and $a p=\lim _{s \rightarrow \infty} s^{-1} \int_{0}^{s} a \exp (t a) d t=$ $\lim _{s \rightarrow \infty} s^{-1}(\exp (s a)-e)=0$.

Example 2.4 ([7] $\mu=0, \xi=1)$. The following conditions on $a \in A$ are equivalent:

(i) The series $\sum_{n=0}^{\infty} a\left(e-a^{2}\right)^{n}$ converges in $A$.

(ii) $a=-p+c$, where $p$ is an idempotent, $c p=p c=p$ and $\left(e-c^{2}\right)^{n} \rightarrow 0$.

(iii) $\sigma(a) \subset M \cup\{0\}$, where $M=\left\{\lambda:\left|\lambda^{2}-1\right|<1\right\}$, and $\mu=0$ is a resolvent point or a simple pole of $a$.

Badiozzaman and Thorpe [1] proved interesting results on summation methods for bounded linear operators on a Banach space (or elements of Banach algebras). 
In [1], condition (ii) in Examples 2.5 and 2.6 below is proved for operators from Condition (I) of Proposition A. The use of Condition (II) provides a considerable simplification and shortening of proofs.

Example 2.5 ([1, Theorem 1]). If $a \in A$ and $\left(a^{k}\right)$ is Abel summable to $p$, then

(i) $p$ is an idempotent,

(ii) 1 is a resolvent point or a simple pole of $a$,

(iii) $(e-a+p)^{-1}$ exists and is equal to $\lim _{t \rightarrow 1-} \sum_{k=0}^{\infty} t^{k}(a-p)^{k}$. If $\left(a^{k}\right)$ is Abel summable, then

$$
\lim _{t \rightarrow 1-}(1-t) \sum_{k=0}^{\infty} t^{k} a^{k}=\lim _{t \rightarrow 1-}(1-t)(e-t a)^{-1}=p .
$$

Write $s(t)=(1-t)(e-t a)^{-1}$ for $t<1$. Then

$$
(e-a) p=\lim _{t \rightarrow 1-}(e-t a) s(t)=\lim _{t \rightarrow 1-}(1-t) e=0 .
$$

So $s(t) p=p$, and $p^{2}=p$. By Theorem 1.2 with condition $(1.7), \lambda=1$ is a simple pole of $a$. Hence the element $-p+a-e$ is invertible by Theorem 1.2. The representation for the inverse of $e-a+p$ can be then obtained as in [1].

Example 2.6 ([1, Theorem 3]). If $a \in A$ and $\left(a^{k}\right)$ is Borel summable to $p$, then the following hold:

(i) $p$ is an idempotent.

(ii) 1 is a resolvent point or a simple pole of $a$.

(iii) $(e-a+p)^{-1}$ exists and is equal to

$$
\lim _{u \rightarrow \infty} \int_{0}^{u} e^{-t} \sum_{k=0}^{\infty} \frac{t^{k}}{k !}(a-p)^{k} d t
$$

Let $\phi(t)=e^{-t} \sum_{k=0}^{\infty} t^{k} a^{k} / k$ !, so that $\phi(t) \rightarrow p$ as $t \rightarrow \infty$. As in [1] it can be deduced that $\phi(t) \rightarrow p a$ as $t \rightarrow \infty$, so that $p a=p$, and ultimately $p^{2}=p$. From $(a-e) p=0$ and Theorem 1.2 with condition (1.7) we deduce that $\lambda=1$ is a simple pole of $a$, and that $-p+a-e$ is invertible. The representation for the inverse of $e-a+p$ is as in [1].

\section{BOUNDED LINEAR OPERATORS}

In the case that $T$ is a bounded linear operator on a Banach space $X$, an isolated spectral point $\mu$ can be characterized by a decomposition of $X$ into a topological direct sum $X=M \oplus N$ satisfying Condition (I) of Proposition A. The direct sum determines and is determined by the spectral projection $P: P X=M$ and $P^{-1}(0)=N$. Mbekhta $[9,10]$ gave an explicit description of the subspaces $M$ and $N$ in terms of $T$ :

$$
M=H_{0}(T-\mu I), \quad N=K(T-\mu I),
$$

where

$$
\begin{aligned}
H_{0}(T)= & \left\{x \in X: \lim _{n \rightarrow \infty}\left\|T^{n} x\right\|^{1 / n}=0\right\} \\
K(T)=\left\{x \in X: T x_{n+1}=x_{n}, T x_{1}=x,\left\|x_{n}\right\| \leq c^{n}\|x\|(n=1,2, \ldots)\right. & \left.\quad \text { for some } c>0, x_{n} \in X\right\} .
\end{aligned}
$$


In general, the spaces $H_{0}(T)$ and $K(T)$ are not necessarily closed, are hyperinvariant under $T$ and satisfy $T^{-n}(0) \subset H_{0}(T), K(T) \subset T^{n} X$ for all $n, T K(T)=K(T)$. For their further properties see $[9,10,11]$.

We give an alternative proof of the implication $(1) \Rightarrow(2)$ in Mbekhta's theorem $[9$, Théorème 1.6] which does not require any use of the local spectral theory or the single valued extension property $[9,11]$. At all times we work only with operators in $L(X)$.

Theorem 3.1 ([9, Théorème 1.6]). Let $X$ be a complex Banach space and $T \in$ $L(X)$. If $\mu \in$ iso $\sigma(T)$ with the spectral projection $P$, then

$$
P(X)=H_{0}(T-\mu I), \quad P^{-1}(0)=K(T-\mu I)
$$

with $H_{0}(T-\mu I) \neq 0$.

Proof. Without loss of generality we may assume that $\mu=0$.

Suppose that $0 \in$ iso $\sigma(T)$ and that $P$ is the spectral projection of $T$ at 0 . By Theorem 1.2, $P T$ is quasinilpotent and $P+T$ is invertible. If $x \in P X$, then $x=P u$ for some $u \in X$,

$$
\limsup _{n \rightarrow \infty}\left\|T^{n} x\right\|^{1 / n}=\limsup _{n \rightarrow \infty}\left\|(T P)^{n} u\right\|^{1 / n}=0,
$$

and $x \in H_{0}(T)$. If $x \in H_{0}(T)$, define $S=(P+T)^{-1}(I-P)$ (the generalized Drazin inverse [8]); then $S T=T S=I-P$, and

$$
\|(I-P) x\|^{1 / n}=\left\|(I-P)^{n} x\right\|^{1 / n}=\left\|S^{n} T^{n} x\right\|^{1 / n} \leq\|S\|\left\|T^{n} x\right\|^{1 / n} \rightarrow 0,
$$

so that $x=P x$. This proves that $P X=H_{0}(T)$. If $x \in P^{-1}(0)$, then the sequence $x_{n}=(T+P)^{-n} x$ satisfies $T x_{1}=x, T x_{n+1}=x_{n}$ and $\left\|x_{n}\right\| \leq\left\|(T+P)^{-1}\right\|^{n}\|x\|$ $(n=1,2 \ldots)$, so that $x \in K(T)$. If $x \in K(T)$, there are $c>0$ and $x_{n} \in X$ such that $T^{n} x_{n}=x$ and $\left\|x_{n}\right\| \leq c^{n}\|x\|(n=1,2, \ldots)$. Then

$$
\|P x\|^{1 / n}=\left\|(T P)^{n} x_{n}\right\|^{1 / n} \leq\left\|(T P)^{n}\right\|^{1 / n} c\|x\|^{1 / n} \rightarrow 0,
$$

and $P x=0$. Hence $K(T)=P^{-1}(0)$.

The other part of Mbekhta's result [9, Théorème 1.6] states that if

$$
X=H_{0}(T-\mu I) \oplus K(T-\mu I)
$$

is a topological direct sum with $H_{0}(T-\mu I) \neq 0$, then $\mu$ is an isolated spectral point of $T$. Schmoeger [11] improved on this result by showing that only $K(T-\mu I)$ needs to be closed. The following theorem generalizes Condition (I) of Proposition A and yields Schmoeger's result [11, Theorem 4] as a special case.

Theorem 3.2. Suppose that $X$ is an algebraic direct sum $X=M \oplus N$, where

(i) $M$ and $N$ are invariant under $T$,

(ii) $(T-\mu I)^{-1}(0) \subset M \subset H_{0}(T-\mu I)$ and $M \neq 0$,

(iii) $N$ is closed and $N \subset(T-\mu I) X$.

Then $\mu \in$ iso $\sigma(T), M$ is closed and

$$
M=H_{0}(T-\mu I), \quad N=K(T-\mu I) .
$$

Proof. Without loss of generality we may assume that $\mu=0$.

Let $P$ be the linear idempotent on $X$ with $P X=M$ and $P^{-1}(0)=N ; P$ commutes with $T$ but need not be bounded. Since $N$ is closed, the operator $T \mid N$ is bounded on $N$; we show that it is bijective on $N$. The injectivity of $T \mid N$ follows 
from the conditions $T^{-1}(0) \subset M$ and $M \cap N=\{0\}$. From $N \subset T X$ and $X=M \oplus N$ it follows that $T \mid N$ is surjective. Since $N$ is a Banach space, $T \mid N$ is invertible in $L(N)$. Hence there is $r>0$ such that $\lambda I-T \mid N$ is invertible in $L(N)$ for all $\lambda$ with $|\lambda|<r$.

Let $\lambda$ satisfy $0<|\lambda|<r$. Let $(\lambda I-T) x=0$. Then

$$
P(\lambda I-T) x=(\lambda I-T) P x=0 .
$$

So $T^{n} P x=\lambda^{n} P x$ for all $n,\|P x\|^{1 / n}=|\lambda|^{-1}\left\|T^{n} P x\right\|^{1 / n} \rightarrow 0$ as $P x \in H_{0}(T)$, and $P x=0$. Similarly $(\lambda I-T)(I-P) x=(\lambda I-T \mid N)(I-P) x=0$, and $(I-P) x=0$. Consequently $x=0$. Conversely, consider $x \in X$. Since $P x \in H_{0}(T)$, the series

$$
\sum_{n=0}^{\infty} \lambda^{-n-1} T^{n} P x
$$

converges. The vector

$$
u=\sum_{n=0}^{\infty} \lambda^{-n-1} T^{n} P x+(\lambda I-T \mid N)^{-1}(I-P) x
$$

is a solution to $(\lambda I-T) u=x$. This proves that $\lambda I-T$ is bijective, and hence invertible in $L(X)$; therefore 0 is isolated in $\sigma(T)$.

It remains to prove (3.2), that is, $M=H_{0}(T)$ and $N=K(T)$ under our assumption $\mu=0$. By hypothesis $M \subset H_{0}(T)$. Also, $N \subset K(T)$ since for each $x \in N$ there are $c=\left\|(T \mid N)^{-1}\right\|>0$ and $x_{n}=(T \mid N)^{-n} x$ satisfying the defining conditions of $K(T)$. By Theorem 3.1, $X=H_{0}(T) \oplus K(T)$, and this completes the proof.

Corollary 3.3. Suppose the space $H_{0}(T-\mu I)$ is nonzero and complemented by a closed T-invariant subspace $N$ satisfying $N \subset(T-\mu I) X$. Then $\mu$ is isolated in $\sigma(T)$, and $N=K(T-\mu I)$.

The following result gives a new characterization of the poles of $T$; we prove only the sufficiency, the necessity follows from [4].

Theorem 3.4. A complex number $\mu$ is a pole of $T$ if and only if for some $q>0$ the space $(T-\mu I)^{-q}(0)$ is nonzero and complemented by a closed $T$-invariant subspace $N$ such that $N \subset(T-\mu I) X$. In this case $N=(T-\mu I)^{q} X$.

Proof. Since $(T-\mu I)^{-1}(0) \subset(T-\mu I)^{-q}(0) \subset H_{0}(T-\mu I), \mu$ is isolated in $\sigma(T)$ by Theorem 3.2, and

$$
(T-\mu I)^{-q}(0)=H_{0}(T-\mu I), \quad N=K(T-\mu I)
$$

by (3.2). If $P$ is the spectral projection of $T$ at $\mu$, then $P X=(T-\mu I)^{-q}(0)$ by Theorem 3.1. So $(T-\mu I)^{q} P=0$, and 0 is a pole of $T$ by the Laurent expansion of Example 2.1. The equality $N=(T-\mu I)^{q} X$ follows from [4, Proposition 50.2].

\section{REFERENCES}

[1] A. J. Badiozzaman and B. Thorpe, A uniform ergodic theorem and summability, Bull. London Math. Soc. 24 (1992), 351-360. MR 93i:47009

[2] R. E. Harte, Spectral projections, Irish Math. Soc. Newsletter 11 (1984), 10-15. MR 86c: 47001

[3] R. E. Harte, Invertibility and Singularity for Bounded Linear Operators, Monographs and Textbooks in Pure and Applied Mathematics vol. 109, Marcel Dekker, New York, 1988. MR 89d:47001

[4] H. Heuser, Functional Analysis, Wiley, New York, 1982. MR 83m:46001 
[5] K. Jörgens, Lineare Integraloperatoren, Teubner, Berlin, 1970. MR 34:8003

[6] J. J. Koliha, Some convergence theorems in Banach algebras, Pacific J. Math. 52 (1974), 467-473. MR 50:8080

[7] J. J. Koliha, Convergence of an operator series, Aequationes Mathematica 16 (1977), 31-35. MR 58:7126

[8] J. J. Koliha, A generalized Drazin inverse, to appear in Glasgow Math. J.

[9] M. Mbekhta, Généralisation de la décomposition de Kato aux opérateurs paranormaux et spectraux, Glasgow Math. J. 29 (1987), 159-175. MR 88i:47010

[10] M. Mbekhta, Sur la théorie spectrale locale et limite des nilpotents, Proc. Amer. Math. Soc. 110 (1990), 621-631. MR 91b:47004

[11] C. Schmoeger, On isolated points of the spectrum of a bounded linear operator, Proc. Amer. Math. Soc. 117 (1993), 715-719. MR 93d:47007

Department of Mathematics, University of Melbourne, Parkville, Victoria 3052, Australia

E-mail address: jjk@mundoe.maths.mu.oz.au 